\title{
Targeting the Immune Checkpoint in Cancer: Is This a Viable Treatment Option for AML?
}

\author{
Steven J Coles* \\ Biomedical Research Group, University of Worcester, UK
}

Received: 酸 March 01, 2018; Published: 非 March 05, 2018

*Corresponding author: Steven J Coles, Biomedical Research Group, Institute of Science and the Environment, University of Worcester, Henwick Grove, Worcester, UK

Abbreviations: AML: Acute Myeloidleukaemia; CBF: Core Binding Factor; mAb: Monoclonal Antibody; MDS: Myelodysplastic Syndrome

\section{Editorial}

The immune suppressive mechanisms displayed by malignant cells are considered a central process in the pathogenesis of cancer. Research in this area has gained significant momentum over the past 20 years, with several immune checkpoints identified, including; CTLA-4, CD200/CD200R, Tim-3/Galectin-9 and PDL1/PD-1 (Figure 1). Whilst characterising the molecular basis of leukaemia for risk stratification remains at the forefront of AML research; this must now extend to understating how these immune checkpoint pathways fit into the equation. A good example of why this is important is to consider CD200 expression level in AML, which is a negative prognostic indicator [1]. CD200 is an immunosuppressive ligand, that when engaged with its receptor CD200R, has the capacity to attenuate T-cell and NK-cell antitumour activity. Interestingly, most cases of CBF AML express high levels of CD200, yet CBF AML performs relatively well clinically. This paradox suggests there is a complex interplay between AML molecular heterogeneity and immune surveillance. Given the recent development and FDA approval of several immune checkpoint therapies, a full understanding of these processes and integration with standard molecular risk stratification is warranted.

The immune checkpoint story is becoming complex for AML, since several studies report that that these immune surveillance pathways function in tandem. For example, the Galectin-9/Tim-3 immune checkpoint has been shown to cooperate with the PD-L1/ PD-1 pathway, which is central in driving CD8+ T-cell exhaustion in AML. Thus targeting both Tim-3/Galectin-9 and PD-L1/PD-1 was required to achieve significant cytoreduction and improved survival in pre-clinical models [2]. Another study illustrated that the CD200/CD200R and PD-L1/PD-1 immune checkpoints are also linked in AML. In this instance, activation of CD200R was sufficient to drive the up regulation of PD-1 on memory CD8 T-cells. Further

analysis relaveled that targeting both CD200/CD200R and PD-L1/ PD-1 immune checkpoints were required to significantly restore memory CD8 T-cell function [3].

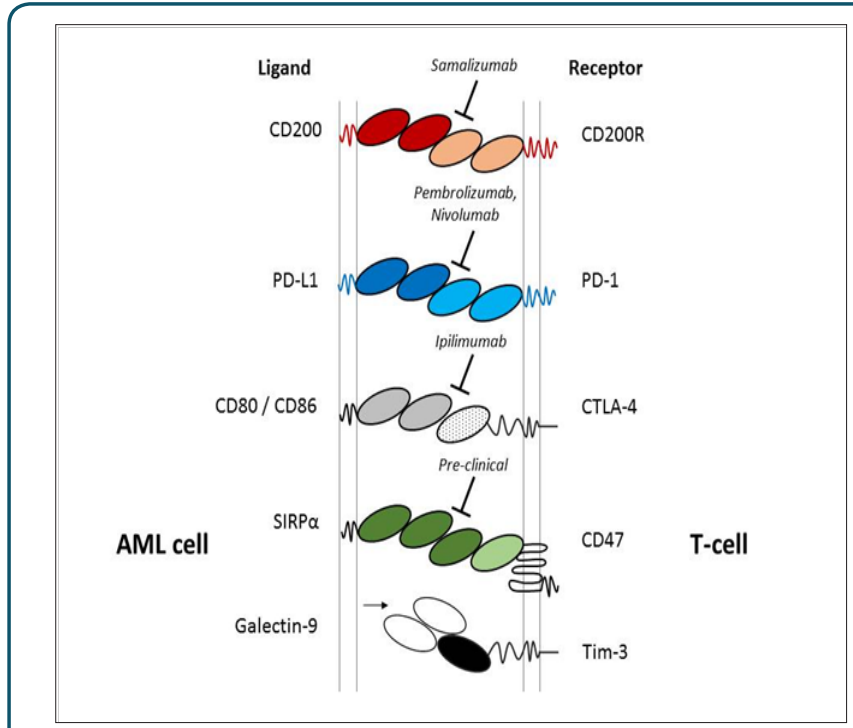

Figure 1: Illustrated are immune checkpoint legends expressed on AML blast cells (left) with the cognate T-cell receptors (right), including; CD200/CD200R, PD-L1/PD1, CTLA-4, CD47 and Galectin-9/Tim-3. Currently clinical trials are exploring the therapeutic potential CD200, PD-1 and CTLA- 4 with the mAb's Samalizumab, Pembrolizumab/ Nivolumab and Ipilimumab respectively. CD47 mAb therapy is at a preclinical stage.

This finding indicates that these immune checkpoints may be important in driving AML relapse. Indeed, this notion is realised in a current phase-II trial (NCT02708641), which is assessing the effects of the PD-L1/PD-1 checkpoint inhibitor 'Pembrolizumab' as 
a post-remission treatment in AML. The potential use of targeting the immune checkpoint in post-remission is also recognised in findings published from a recent phase-IB/II study involving the PD-L1/PD-1 checkpoint inhibitor 'Nivolumab'. The report shows that when used in combination with azacytidine for relapsed AML, Nivolumab showed an improvement in prognosis and increased numbers of effector CD8 ${ }^{+}$T-cells [4]. Given that the PDL1/PD-1 checkpoint functions in combination with other immune checkpoints, the question now is to understand whether targeting a combination of these pathways in AML performs well clinically.

To this end, results from a current phase-II trial (NCT02530463) targeting PD-L11/PD-1 with Nivolumab in combination with the CTLA-4 inhibitor 'Ipilimumab' in MDS are eagerly awaited. As are the next generation of therapeutic mAb's such as 'Samalizumab' (Alexion Pharmaceuticals), which is designed to target the CD200/ CD200R checkpoint. The potential for immune checkpoint therapy in AML is clearly evident, however the interplay between these pathways needs full appreciation and placed into context with molecular stratification and standard therapy (Figure 1).

\section{References}

1. Tonks A, Hills R, White P, Rosie B, Mills KI, et al. (2007) CD200 as a prognostic factor in acute myeloid leukaemia. Leukemia 21: 566-568.

2. Zhou Q, Munger ME, Veenstra RG, Weigel BJ, Hirashima M, et al. (2011) Coexpression of Tim-3 and PD-1 identifies a CD8 ${ }^{+}$T-cell exhaustion phenotype in mice with disseminated acute myelogenous leukemia. Blood 117: 4501-4510.

3. Coles SJ, Gilmour MN, Reid R, Knapper S, Burnett AK, et al. (2015) The immunosuppressive ligands PD-L1 and CD200 are linked in AML T-cell immuno suppression: identification of a new immunotherapeutic synapse. Leukemia 29: 1952-1954.

4. Daver N, Basu S, Garcia Manero G, Cortes JE, Ravandi F, et al. (2016) Phase IB/II Study of Nivolumab in Combination with Azacytidine (AZA) in Patients (pts) with Relapsed Acute Myeloid Leukemia (AML). Blood 128: 763.

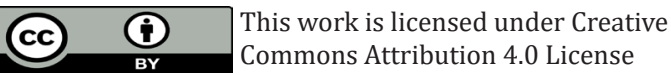

To Submit Your Article Click Here:

Submit Article

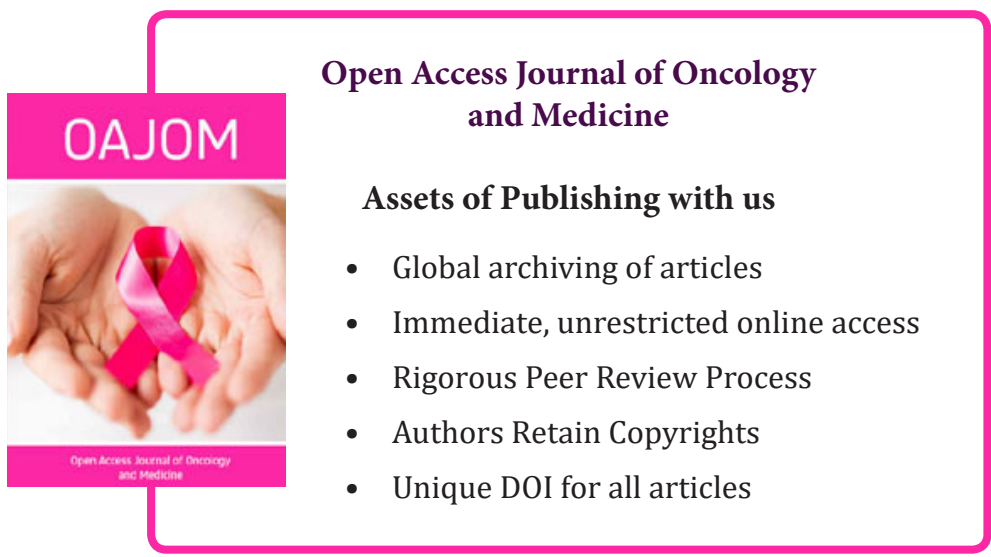

